INTRODUCTION

\title{
La fabrique des politiques publiques co-construites : idéologie et pratiques innovantes
}

\author{
> Madina Rival ${ }^{a}$ et Jean-Claude Ruano-Borbalan ${ }^{b}$ \\ ${ }^{a}$ Cnam-Lirsa \\ ${ }^{b}$ Cnam-HT2S
}

L'analyse des politiques publiques (Fischer, Miller et Sidney, 2007; Boussaguet, Jacquot et Ravinet, 2015) est une thématique en renouvellement notamment - mais pas uniquement- en Sciences politique ou en sciences de gestion. On trouve aussi, de l'urbanisme aux Sciences and technologies studies en passant par la sociologie, le design ou l'histoire, de nombreuses analyses sur les jeux d'acteurs, les questions d'expertise ou de mobilisation, les formes de gouvernance, les institutions. Si le champ de l'action publique n'est pas du tout nouveau et constitue même l'une des préoccupations majeures des sciences sociales le point de vue s'est déplacé. On ne prendra qu'un exemple au travers de la réflexion sur le développement de l'économie territoriale et les systèmes d'innovation où convergent des préoccupations de sciences de gestion, d'économie, de management public. On constate que la réflexion se porte là sur l'articulation des différentes échelles d'intervention et l'intrication des formes de l'action publique (Boutillier et al., 2014). Les nouvelles échelles explorées pour l'analyse de l'intervention ou de l'action (échelles territoriales, managériales, organisationnelles) sont mises en regard de conception plus habituelle d'analyse, comme par exemple dans le cas spécifique mais emblématique des systèmes nationaux ou régionaux de recherche d'innovation étudiés grâce à des visions disciplinaires multiples (Ortiz, 2014; Pestre, 2013). La question de l'importance des échelles locales et individuelles, tant pour l'analyse dans les approches praxéologiques que pour l'action et d'intervention dans certains champs disciplinaires. Ces questions sont aujourd'hui singulièrement posées dans le cadre de la très grande vague idéologique à l'œuvre autour de la promotion des visions de l'action publique ou de l'économie en termes « d'innovation » (Godin, 2015) et d'accompagnement du développement économique par l'incitation et «l'encapacitation».

La spécificité de notre point de vue est de s'intéresser plus particulièrement à la mise en place des politiques publiques, aux mécanismes et stratégies d'acteurs qui interviennent dans le processus d'élaboration, aux mécanismes politiques et à la réalisation 
de l'action publique. C'est ce qu'il est désormais convenu de nommer « la fabrique des politiques publiques ». Cependant, alors que Zittoun (2013) s'intéresse par exemple à la « fabrique politique des politiques publiques », c'est davantage leur construction dans le cadre d'interactions et mobilisations stratégiques et pragmatiques qui nous intéressent.

L'un des enjeux majeurs actuels en la matière réside dans la multiplicité des acteurs et des points de vue qui participent à la fabrique de ces politiques publiques. Les « fabricants privés » des politiques publiques peuvent être à but lucratif (entreprises) ou non (associations, citoyens). C'est ce que nous appelons une construction plurielle. Au-delà des « co » décisions au sein de la sphère publique, le fait marquant réside dans la fin du monopole des décideurs publics qui fait place à l'intervention d'un nombre de plus en plus important d'acteurs privés. Des valeurs, à la gouvernance, en passant par la mise en œuvre, ce fait stylisé questionne la fabrique des politiques publiques. En contrepoint de la littérature consacrée régulièrement aux discours et critiques du «new public management » (Chanut et al., 2014), nous souhaiterions nous attacher à comprendre les processus de négociations et d'expertises multiples mobilisés dans des processus de définition comme de mise en œuvre de politiques publiques. La visée est praxéologique et le but de mieux connaître «l'agir en management public ». Le projet est en premier lieu de décrire des pratiques diverses pour comprendre les régularités ou les singularités (1). Deux thèses se répondent dans le développement qui suit. Pour la première, la participation plurielle à la fabrique des politiques publiques peut se révéler source d'innovation et d'efficacité (2). Pour la seconde, l'intervention de nouvelles parties prenantes dans les processus de définition et d'implémentation des politiques publiques appelle aujourd'hui une distance critique visà-vis de ces nouveaux dispositifs (3). Nous proposons ensuite un résumé problématisé des articles retenus dans le présent numéro spécial (4).

\section{La co-construction des politiques publiques : un fait reconnu en sciences humaines et sociales}

La fabrique des politiques publiques est désormais un objet hybride (Christensen et Laegreid, 2010), notamment en raison de l'intervention sur le terrain d'acteurs privés que l'on désignera dans leur globalité par les termes «entreprises » et « société civile » ou « citoyens ». Cette intervention est aujourd'hui acceptée par les administrations publiques (Bérard, 2013). Elle n'est plus analysée comme une simple influence, mais comme une véritable co-construction (Rival et Sémo, 2014). Ce point est particulièrement étudié dans les politiques liées au social, au développement urbain, aux questions environnementales et technoscientifiques (santé, risques, etc.) et fait l'objet d'une littérature abondante.

Dans une période de «vide stratégique » (Baumard, 2012) pour les entreprises et de restriction budgétaire dans le domaine public, l'influence des acteurs privés dans les politiques publiques est devenue une réalité assumée. Pour les entreprises, depuis toujours, la stratégie ne repose pas seulement sur une approche interne du développement, mais prend en compte et tente de peser en sus des aspects de marché, sur l'environnement normatif, les institutions de formation, les politiques centrales au plan social et autre. Cependant, au cours du $\mathrm{XX}^{\mathrm{e}}$ siècle, le rôle central et accru de l'État, dans tous les pays développés, a amoindri la capacité d'intervention des entreprises dans les politiques publiques. L'histoire industrielle 
montre par exemple qu'en matière de formation des ingénieurs et de développement de l'innovation, le rôle des entreprises, particulièrement prégnant au $\mathrm{XIX}^{\mathrm{e}}$ siècle, a singulièrement reculé au profit d'un monopole étatique au cours du $\mathrm{XX}^{\mathrm{e}}$ siècle, pour d'ailleurs revenir au premier plan depuis quelques décennies (Bertilorenzi, Ruano-Borbalan et Le Coq, 2017). On peut reprendre, dans la perspective d'une entreprise ouverte (Baron, 1995), l'existence d'un «non market environment » (environnement hors marché) qui inclut les forces sociales, politiques et légales extérieures au marché, en interaction avec les intérêts privés. Des stratégies hors marché peuvent être définies de manière générale. Elles comprennent pour les firmes les stratégies sociétales (responsabilité environnementale et responsabilité sociale de l'entreprise, désormais notée RSE, par exemple) et les stratégies politiques (lobbying notamment) destinées aux décideurs publics, mais aussi de manière plus systémique, les actions de communications en direction de multiples parties prenantes de la société civile, de la recherche ou des collectivités territoriales. Toutes ces formes d'intervention impactent les politiques publiques. Elles se comprennent comme toutes les activités destinées à modifier directement ou indirectement les processus d'élaboration, d'application ou d'interprétation de mesures législatives, normes, règlements et plus généralement décisions des pouvoirs publics (Rival et Chanut, 2015). Lawton et al. (2014) montrent bien que ces stratégies (politiques ou autres) sont indispensables à la performance des entreprises.

Par ailleurs, et ce depuis près d'un demi-siècle à la faveur des crises sanitaires, problèmes environnementaux ou autres, on constate l'intervention de plus en plus identifiée de ce que la littérature de sciences politiques, reprise dans d'autres disciplines nomme la « société civile» (McCall, 2007). Les citoyens, de manière individuelle ou par le biais d'associations, interviennent directement et ne sont plus simplement des objets du management public (Michel, 2005) mais également des sujets. Au-delà des mouvements sociaux (Revilliard, 2016), les théories de la réception de l'action publique montrent notamment des « ressortissants [destinataires de la politique publique] coproducteurs de l'action publique » (Warin, 1999). Les secteurs de la formation et de la santé sont des terrains fertiles en la matière. C'est ainsi que la question de l'université est régulièrement l'objet de recherches (Mazeaud, 2013). L'un des lieux majeurs de cette intervention, fortement interrogée par la littérature des Science and technology studies, réside dans l'interface sciences, techniques, économie et politiques publiques. Globalement et depuis la fin du siècle dernier on constate des processus puissants de standardisation de la production scientifique ou industrielle et des procédures administratives. Cette optimisation de plus en plus efficace des interfaces entre les sciences, les politiques publiques et le monde économique, s'exprime essentiellement sous la forme de processus d'innovations linéaires, aussi bien industrielles que de gestion ou managériaux. Ces processus s'appuient, d'une part, sur une gestion de plus en plus complexe de portefeuilles de propriétés intellectuelles, et d'autre part, sur une diversité d'outils et de dispositifs allant du transfert de technologie à la création de pépinières d'entreprises. L'effet massif et généralisé est indéniable, mais il demeurait cantonné dans les sphères économiques strictes. Depuis les années 2000, les limites de ce modèle sont apparues pour de multiples raisons, notamment liées à la faible prise en compte des externalités négatives de ce modèle de développement : dégradation de l'environnement (émergence d'une conception systémique d'anthropocène), changement climatique, problèmes sanitaires, mais aussi sociaux avec la montée de l'individualisme entraînant une crise démocratique des sociétés contemporaines. 
D'un côté, on assiste à un élargissement sans précédent du champ de l'innovation qui englobe les sphères économiques, politiques et autres, largement appuyé aujourd'hui par les recherches scientifiques. De l'autre, on constate une montée (au moins idéologique et d'intention) de formes plus diffuses de l'innovation, plutôt « ascendantes », qui rencontrent et irriguent depuis deux décennies des volontés d'amélioration des fonctionnements administratifs ou de la puissance publique. Ces formes sont devenues des références de l'activité dite « citoyenne », notamment au travers de l'innovation sociale ou des volontés et formes multiples de «participation » et engagement : fab-labs et living labs, mobilisations et controverses technoscientifiques, sciences et recherches participatives, innovations sociales et territoriales, boutique des sciences, etc.

La question de l'échelle de ces multiples points de vues est interrogée. Les territoires, propices à l'expérimentation (Durance, Godet et Mousli, 2010) sont parfois qualifiés de « hautement citoyens » (Le Coz, 2015). Des réseaux d'acteurs et des outils existent pour favoriser cette démocratie locale. Il va sans dire qu'internet et les réseaux sociaux catalysent l'intervention citoyenne dans la fabrique des politiques publiques en favorisant l'accès à l'information et la capacité de mobilisation de chacun (Colin et Verdier, 2015). La question très actuelle des lanceurs d'alerte n'est pas loin. Plus généralement, les potentialités et les points de vigilance de cette fabrique de politiques publiques en contexte « datacentrique » sont examinés par les praticiens comme les chercheurs (Carmes et Noyer, 2014).

\section{La participation des acteurs privés, une source d'innovation publique?}

Ces fabriques multi-acteurs sont une réalité aujourd'hui que les « décideurs politiques disent utiliser pour répondre à la complexité de leur environnement décisionnel » (Bérard, 2013 : 51). Plus précisément, en termes de valeur ajoutée, cette « co-construction » est de plus en plus analysée en termes d'innovation publique.

L'innovation publique est définie sur le site internet du Secrétariat général pour la modernisation de l'action publique ${ }^{2}$ comme « une administration en mouvement, qui promeut l'innovation dans les services publics, de la conception à la mise en œuvre des politiques publiques ». Elle est appréhendée dans la littérature gestionnaire notamment sous le prisme de l'innovation managériale appliquée au secteur public notamment au niveau des outils et de l'organisation (Marin et al., 2016). De nombreuses recherches qui mettent en exergue la spécificité de l'innovation publique managériale se sont développées. Ces analyses « tendent à confirmer l'idée d'un management public « hybride » ou intermédiaire qui procède à la fois de transpositions (notamment en ce qui concerne la finalité de la démarche, les conditions d'implantation ainsi que l'importance d'un certain style de leadership) mais aussi d'innovation et de différenciation (voire de démarcation) par rapport au management privé » (Favoreu, 2008 : 2). Ces travaux mettent notamment en évidence l'importance du « leadership politique » et du temps dans les processus de changement du secteur public.

Les notions d'influence et de plus en plus de «co-construction » amènent à réinterroger cette définition managériale de l'innovation publique, pour s'intéresser davantage aux processus. Il ne s'agit plus seulement de laisser simplement les agents « street level»

2 http://www.modernisation.gouv.fr/la-semaine-de-linnovation-publique/presentation-de-la-semainede-linnovation-publique, consulté en juillet 2017. 
(agents d'exécution) s'adapter intelligemment aux demandes des usagers dans un processus d'innovation des petits pas ( « nudge ») comme cela a longtemps été étudié (Dubois, 2012). Il s'agit dans les intentions, d'aller vers des démarches volontaristes de participation et d'expertise interactionnelle des diverses parties prenantes (notamment de la société civile) qui peuvent générer de l'innovation incrémentale, et du moins l'espère-t-on, radicale.

Il ne s'agit pas pour nous de revenir sur le volontarisme des pouvoirs publics pour générer des écosystèmes créatifs (Horvath et Dechamp, 2016) mais bien de s’interroger sur les processus réels de «fabrique » de ces politiques en tant qu'ils peuvent être considérés comme forme d'innovation. Nous nous inscrivons dans les travaux les plus récents sur l'innovation publique qui mettent en avant d'avantage la spécificité du processus plus que le résultat (Ewango Chatelet, 2017).

Dans la sphère économique et entrepreneuriale, la question des processus dits « collaboratif » et ou « innovants » a été examinée depuis longtemps (Alcouffe et D'Armagnac, 2009). Au début des années 2000, certains comme Chesbrough (2003) ont prôné une innovation collaborative puissante, basée sur les partenariats lors des différentes étapes du processus : recherche fondamentale (technologie externe acquise par achat ou alliance), recherche appliquée (licence, essaimage, vente). De même, dans le secteur public, Chantelot et Errami démontrent que la métropole brestoise a pu construire une ville créative grâce à trois niveaux de participation (repris de Cohendet et al., 2010) : les décideurs publics arrivent en troisième position au sein des organisations constituées dites « upperground». L'innovation publique est permise par l'intervention de deux niveaux inférieurs de participation : les créatifs (chercheurs, entrepreneurs, incubateurs) dits « underground » et les réseaux qui diffusent ces innovations aux pouvoirs publics dits « middleground ». On distingue ici l'innovation d'exploration (les parties prenantes) de l'innovation d'exploitation (les décideurs publics). La notion de « design de service public » très en vogue depuis moins de dix ans pour générer de nouvelles « formes d'innovation publique » se caractérise en réalité par différents modes de co-construction de politiques publiques (Weller et Pallez, 2017 : 47) : «Consultation d'habitants d'un territoire pour l'aménagement de l'espace public » ou «Co-confection d'interfaces numériques ou documentaires pour équiper la décision » par exemple.

\section{La co-construction comme idéologie et comme stratégie d'acteurs}

Une question, fondamentale aujourd'hui dans l'analyse des politiques publiques, tourne autour des questions liées à l'expertise, aux normes, à l'évaluation qui s'incarnent notamment dans une interrogation forte sur les instruments intellectuels, organisationnels, techno-scientifiques mobilisés (Halpern et al., 2014). L'exemple connu de la normalisation comptable internationale est illustratif. Les normes sont prises en charge par les acteurs privés qui disposent aujourd'hui de l'expertise et des ressources pour les rédiger (Rival et Vidal, 2013). Ces normes fabriquent des politiques en orientant progressivement ce qu'il est possible de faire et concourent à l'action publique dont les frontières deviennent floues. Une telle situation, d'un point de vue juridique, n'est pas historiquement novatrice, puisque dans la tradition romano-occidentale du droit c'est précisément le contrat et le procès qui constitue la source des interactions aux échelles locales ou individuelles (Bretone, 2015). Le 
renforcement historique de l'État-nation et de sa régulation depuis quelques siècles paraissait tendre vers un englobement complet et une sujétion de la normativité. Or il semble que l'on assiste plutôt à une réémergence de régulations par les contrats ou les acteurs, et à la mise en œuvre de formes plus hybrides, notamment sous l'effet des technologies de communication interactive en réseau (Web), et des exigences de participation et de l'intervention citoyenne ou des parties prenantes. La question de la propriété intellectuelle et des brevets illustre l'un des problèmes que l'intervention dite « collaborative » permettrait possiblement de résoudre selon certains auteurs. Le nombre de dépôts pour protection par brevet est en croissance exponentielle dans le monde et le retard des institutions de certification de plus en plus important, menaçant les fonctionnements mêmes de l'économie et de l'exercice de la propriété industrielle et intellectuelle. Un débat a lieu pour la mise en œuvre de «peer to peer patents », brevets qui seraient le fruit de la validation et légitimation par des usagers et experts via des processus en ligne (Noveck, 2006).

L'un des points essentiels à interpréter dans le moment actuel de renforcement d'une vision participative et coopérative de l'action publique est celui de la légitimation des cadres et réalisation des politiques publiques, tant du point de vue strictement démocratique (Rosanvallon, 2008) que des sources de la validation, issues des savoirs mobilisés, en l'occurrence de l'expertise scientifique ou scientifiquement référée (Ruano-Borbalan, 2014).

L'analyse de la production des politiques et des connaissances ou normes en situation, par les acteurs, permet l'interprétation des mutations de l'autorité et de la légitimité de l'action et du management public et in fine des formes du « gouvernement » (Pestre, 2014). On le mesure de multiples manières et particulièrement dans les transformations des liens entre puissances publiques et associations. Si le développement associatif est vu par la littérature sociologique comme un puissant facteur de lien social au travers du développement du « capital social » des individus, il est également apprécié comme moyen historique de développement de la démocratie. La situation actuelle est plutôt considérée par certains comme un moment de faiblesse de la capacité d'intervention de la société civile, comme de l'État ou des acteurs publics face au rouleau compresseur supposé du « néo-libéralisme » (Laville et Salmon, 2015).

En fait, si l'on apprécie plutôt la systémique globale de l'action publique, les questions sont singulièrement complexes et les firmes ne sont pas forcément dominantes dans la mise en œuvre régulatrice. Les questions sont à voir secteur par secteur, situation par situation. C'est plus de multiplicités de stratégies et configurations de pouvoirs qu'il s'agit, dans lesquelles les formes d'action ou d'expertises mobilisées s'appuient sur des légitimités elles aussi multiples. Ni l'autorité de la Science ou de l'État «per se » ne sont acceptables comme telles. Les différents acteurs et parties prenantes cherchent des moyens d'action et de pesée en convoquant toutes les formes d'expertise ou de légitimation acceptables. C'est là que l'on verra les concurrences d'expertise se développer (Collins, 2014; Goreman, 2010). C'est là aussi que l'on cherchera à s'appuyer sur les validations par le nombre ou des formes renouvelées d'autorité par la «collaboration » ou la «participation », notamment liée à l'usage des nouvelles technologies et des réputations ou autorités qui s'y sont affirmées (Broudoux, 2007).

On peut comprendre l'intervention des parties prenantes dans les processus (souvent locaux) de participation et de « co-construction » des politiques publiques comme l'expression d'une idéologie globale née face à une transformation de la légitimation démocratique et aux conséquences nationales de transformations internationales en matière normative 
ou d'action publique (Benyekhlef, 2015). On peut également, et ce n'est nullement contradictoire, la penser comme une ressource argumentative et stratégique pour les différents acteurs. L'analogie avec les idéologies identitaires (religieuses, ethniques ou autres) fort étudiées par la littérature en relations internationales ou en sociologie politique pourrait être faite : il est toujours délicat de privilégier une interprétation par rapport à une autre, tant les acteurs sont capables de mobiliser les référents idéologiques pour des fins « utilitaristes » et stratégiques (Heinich, 2017). On peut cependant raisonnablement affirmer que les processus de «co-construction» et interactions stratégiques entre acteurs parties prenantes des politiques publiques, plus ou moins sollicités institutionnellement, participent directement de la mise en œuvre d'un management public. On le sait, l'une des modalités de la légitimation de l'action, qu'elle fut publique ou non, est le respect de rituels et procédures jugés comme légitimant (Scharpf, 2010). Ainsi, les nouvelles modalités de construction des politiques publiques au plus près et en collaboration, peuvent être considérées comme une des modalités de légitimation du management public en situation de transformation et de défiance de formes antérieures de l'action publique. Le management public «tantôt considéré comme un art, tantôt vu comme une science, (il) peut tout aussi bien être considéré comme un mouvement ou comme un outil de légitimation. » (Gibert, 2008 : 7). Entre acteurs publics et acteurs privés, on peut bien entendu se demander «qui » est légitime dans la fabrique des politiques publiques? Mais la question ainsi formulée renverrait à une compréhension de l'action publique en termes immuables, ce qu'elle n'est, ni ne fut jamais. La voie la plus prometteuse est ainsi de ne pas répondre à cette question, pour considérer la dynamique en cours comme une tentative de définition de formes renouvelées d'autorité et de légitimation, dans laquelle la validité de l'expertise est absolument cruciale et constitue le lieu focal des désaccords politiques. Une telle approche pose cependant une question forte : comment construire des outils et techniques pour le contrôle et l'évaluation des politiques publiques co-construites? Objet d'enjeux politiques et praxéologiques majeurs, ces questions ne se résolvent pas on le sait par la généralisation ad nauseam du NPM (New Public Management) fondé sur des indicateurs construits eux sur des modèles antérieurs de conception de l'intervention. Les approches d'aménagement du territoire ou de prospective, aujourd'hui battues en brèche au profit de notions issues de disciplines et action politiques comme l'urbanisme, le design ou de la démocratie participative pourraient illustrer largement ces interrogations.

En conclusion, au niveau local comme international, on constate la prégnance d'une interrogation sur la frontière entre les secteurs publics et privés. Cette interrogation n'est pas nouvelle puisque dès 1980, Alain Burlaud et Romain Laufer affirmaient déjà au sujet du management public : «Il est le résultat de la crise que connaît la délimitation entre secteur public et privé », mais elle est aujourd'hui renouvelée, par la force des approches managériales convergentes fondées notamment sur la créativité/attractivité organisationnelle, territoriale ou/et individuelle. De la complexité des pratiques publiques (Chanut, 2004) naissent différentes politiques de terrain comme celles menées en matière de formation, d'éducation et de santé. Il est tentant de se demander quelles rationalités et quelles frontières sont à définir pour optimiser l'action, mais ce que l'on a dit plus haut plaide pour une vision plus « configurationnelle » qui sied avec les grandes représentations collectives du début du XXI $\mathrm{XI}^{\mathrm{e}}$ siècle, autour des capacités supposées des acteurs divers de coopérer ou d'obtenir des compromis. Il demeure que les formes institutionnelles et les normes juridico 
administratives sont loin d'avoir disparu et qu'au contraire il convient de penser la situation comme une dialectique entre des stratégies utilitaristes et forces et négociations au plan local ou subsidiaire, et des formes de régulations autour des biens communs, fussent-ils mondiaux (Delmas-Marty, 2017). Les politiques publiques ne sont plus, en tout cas plus seulement, l'apanage de l'administration ou de la loi, mais résultent de multiples interventions et arrangements qui s'imposent dans le cadre de cette nouvelle fabrique des politiques publiques : entre hétérogénéité et homogénéité.

Quel que soit l'angle de la réflexion, on constate la prégnance dans les politiques publiques et l'économie, on l'a dit, de la préoccupation d'innovation, liée en premier lieu au développement d'un mode de production techno-scientifique dominant et particulièrement efficace au plan productif, comme au développement d'initiatives « d'innovation sociale » multiformes (Ruano-Borbalan, 2017). Depuis une dizaine d'années et en lien avec les politiques d'orientation européennes, l'une des grandes postures a consisté à mettre en place un système national de recherche et d'innovation renforcé et « cohérent» (Lesourne et Randet, 2013). Cette politique, déclinée en multiples actions et formes d'incitation ou coordination, est destinée à forcer la croissance et maintenir l'économie dans la compétition entre nations dites « de la frontière », au plan recherche et innovation (Berger, 2013). La « co-construction » s'observe bien dans le domaine des politiques publiques de recherche et passe par une volonté de développement d'une « science participative » et de développement de l'innovation alliant sciences, technologies et usages. Sont ici privilégiées les notions d'interfaçage entre différents champs impactés par la transformation de la production techno-scientifique : de la réaction des populations face à des risques ou controverses scientifiques, aux contraintes technologiques ou scientifiques, jusqu'aux usages économiques, entrepreneuriaux ou « citoyens ». Il est à noter que les analyses sont divergentes, selon les fondements disciplinaires, axiologiques voire prescriptifs. Les sciences de gestion, l'économie de l'innovation peuvent privilégier l'analyse (souvent en vue de prescription) des formes de gouvernance institutionnelles, des grappes d'entreprises et pôles de compétitivité techno-scientifiques (orientés pour une part autour de discours de la promesse entrepreneuriale et des «start-ups »). L'approche socioanthropologique des sciences et techniques, critique pour l'essentiel, privilégie, elle, clairement la capacité des acteurs individuels ou associatifs de peser sur les choix scientifiques, voire la construction des savoirs. Dans un entre-deux, on trouve plus typiquement des approches urbanistes, architecturales, de design, dont la visée prescriptive, liée à des maître d'œuvre ou logiques de financement macro-économiques, entrepreneuriales ou d'aménagement territorial, est patente. Ces approches sont orientées clairement et de plus en plus sur l'hypothèse du bien-fondé de la participation et de l'intervention des usagers : en syntonie avec la vision idéologique dominante dans les pays européens, autour de l'importance de l'action et de la maîtrise « citoyenne ».

\section{Présentation des articles sélectionnés}

Un résumé problématisé des huit articles retenus dans ce numéro spécial sur les vingtdeux reçus est présenté ici. Soulignons de prime abord que les champs sont divers : sciences de gestion, sociologie, urbanisme, science politique et les points de vue différents : local, territorial, national, européen, international. Ces recherches portent sur différents types 
d'acteurs de la fabrique : externes comme les citoyens, les entreprises, les associations ou des méta-organisations, mais également internes au secteur public comme les différentes collectivités voire internes aux organisations publiques comme les fonctionnaires. Les articles sélectionnés ont en commun de porter la tension entre dispositif incantatoire et potentialités d'innovation publique en présentant des cas concrets.

Notons que le concept de fabrique des politiques publiques est large et inclut l'amont (conception) comme l'aval (mise en œuvre par les sous entités, collectivités, fonctionnaires) par laquelle la politique est en réalité redessinée (re-designed). C'est la dichotomie que nous avons choisie pour présenter succinctement les articles de ce numéro spécial : quatre se situent en amont de la fabrique (que l'on pourrait qualifier sur le modèle de la recherche et développement comme la «fabrique fondamentale ») et quatre en aval (ou «fabrique appliquée » pour filer la métaphore de la R\&D).

\section{La fabrique fondamentale}

Cappelletti et Khenniche présentent une fabrique en amont de politiques publiques en s'intéressant classiquement au couple politico administratif au sein d'une commune. La démarche de recherche est originale puisqu'elle repose sur une recherche-action dans le cadre d'une politique visant la performance socialement responsable.

Berkowitz et Souchaud présentent une fabrique en amont de politique publique moins attendue : ou comment des méta-organisations (organisations d'organisations) privées vont influencer la régulation du secteur de la finance participative (crowd funding) via une ordonnance et ses décrets d'application. Au-delà des traditionnelles « corporate political studies », cet article propose une nouvelle vision de la dynamique des entreprises privées en matière de politique publique.

Pour Fausshauer et Zadra-Veil, c'est un autre type d'organisation ad hoc qui se situe en amont des politiques publiques : le living lab qui réunit entreprises, associations, citoyen et éventuellement les pouvoirs publics. Ici apparaît la notion de proximité et de territoire qui traverse un certain nombre d'articles de ce numéro spécial. Ici également il s'agit d'une méta-organisation (une structure hybride) dont l'un des objectifs est l'innovation sociale ou publique. La notion de politique est entendue au sens large (le cas concerne différents aspects locaux dont une politique de sensibilisation santé). L'article met en évidence des limites comme la difficulté de la co-construction entre institutions publiques et citoyens (inégaux en pouvoir et légitimité comme en attentes) et le partage de la valeur.

Fenker et Zetlaoui-Léger nous proposent une fabrique entre amont et aval, entre dispositif anté performatif et pratique innovante également. L'État français met en place un dispositif d'incitation aux écoquartiers dans le cadre de l'implémentation de la politique nationale de développement urbain... et pourtant l'implémentation nationale au niveau local via la participation citoyenne se révèle génératrice d'innovation, notamment en matière d'ingénierie de projet. Cet article présente en outre un cas multi-acteurs : ministères, collectivités (insiders)/société civile (outsiders).

\section{La fabrique appliquée}

Fraisse propose le panorama d'une intervention en aval classique : celle des associations. Cet article met en avant les facteurs clés de l'apparition de cette co-construction que nous pourrions mettre en parallèle avec la notion bien connue désormais d'alignement stratégique dans les entreprises. Ici, il apparaît que la fabrique collective de la politique 
publique sera mise en avant lorsqu'il y a, d'une part une cohérence entre le sens de la politique induite et les valeurs portées par la participation, et d'autre part « un déficit de ressources politico-administratives ».

Kustosz en revanche s'intéresse à la participation d'un acteur privé plus original et bien particulier : l'entrepreneur dans le cadre d'une diffusion de l'innovation appelée » découverte entrepreneuriale ». Le cas étudié est celui de l'implémentation d'une politique européenne à l'échelle régionale. Cette co construction est source d'innovation puisque c'est le propos du dispositif mais aussi via une externalité positive puisqu'une gouvernance publique innovante apparaît : basée sur l'expertise issue de l'expérience elle est caractérisée par « une dynamique bi-rectionnelle et itérative » (top down et bottom up). L'existence d'un réseau et la territorialité sont aussi considérées dans ce cas comme des vecteurs d'innovation publique, la lourdeur du dispositif européen et national pouvant être un frein.

Mériade, Nautré, Rochette et Talbot décrivent les effets positifs de la proximité pour une bonne exécution de la politique publique car elle favorise une interaction positive entre : personnels, organisation et politiques via le triptyque : confiance, contrôle, légitimité. Cet article introduit la dimension de la pluralité des acteurs publics (insiders et ousiders) qui se retrouve par exemple dans l'article de Savard et al.

Boily et Savard s'intéressent au rôle d'un insider : le fonctionnaire (et plus précisément les interactions entre fonctionnaires). Il s'agit d'un focus métier et d'un contrepoint international sur la question qui nous occupe (le cas du ministère fédéral canadien de l'environnement). L'innovation publique dans ce cadre proviendrait de la « créativité » des individus fonctionnaires qui détourneraient les règles pour mieux « satisfaire les raisons d'existence de l'organisation » publique. 


\section{Bibliographie}

Alcouffe C. et D'Armagnac S., 2009. Analyses et transformations de la firme. Une approche pluridisciplinaire. La Découverte, Paris.

Baron D.P., 1995. Business and its Environment. Prentice Hall, Upper Saddle River.

Baumard P., 2012. Le vide stratégique. CNRS Éditions, Paris.

Benyekhlef K., 2015. Une possible histoire de la norme : Les normativités émergentes de la mondialisation. Themis, Montréal.

Bérard C., 2013. Les démarches participatives en matière de politiques publiques : le cas de la propriété intellectuelle des innovations biotechnologiques. Politiques et Management Public 30 (1), 51-73.

Berger S., 2013. Making in America, from Innovation to Market. MIT Press, Cambridge.

Burlaud A. et Laufer R., 1980. Management public, Gestion et légitimité. Dalloz, Paris.

Boussaguet L. Jacquot S. et Ravinet P., 2015. Une «French touch» dans l'analyse des politiques publiques. Presses de Sciences Po, Paris.

Boutillier S. et al., 2014. Principes d'économie de l'innovation, Réseau de Recherche sur l'innovation. Peter Lang, Bruxelles.

Bretone M., 2015. Histoire du droit romain. Éditions Delga, Paris.

Broudoux E., 2007. Construction de l'autorité informationnelle sur le web In Skare R., Windfield Lund N., Varheim A., A Document (re)turn (Contributions from a Research Field in Transition). Peter Lang/ Europäischer Verlag der Wissenschaften, Bern, 265-278, mars 2007.

Carmes M. et Noyer J.M., 2014. Devenirs urbains. Presses des Mines/ParisTech, Paris.

Chanut V., 2004. L'État didactique. L'Harmattan, Paris.

Chanut V. et al., 2014. Nouvelles idées sur le management public, un champ en construction? communication à la Troisième conférence de l'Association internationale pour la recherche en management public, AIRMAP, Aix en Provence, mai.

Colin N. et Verdier H., 2015. L'âge de la multitude, entreprendre et gouverner après la révolution numérique. Armand Colin, Paris.

Chantelot S. et Errami Y., 2015. Stimuler le potentiel créatif des environnements urbains. Revue Française de Gestion 250, 139-158.

Chesbrough H., 2003. Open Innovation - the new Imperative for Creating and Profiting from Technology. Harvard Business School Press, Harvard.

Christensen T. and Laegreid P., 2010. Complexity an hybrid public administration - theorical and empirical challenges. Public Organization Review 11 (4), 1-17.

Cohendet P., Grandadam D. and Simon L., 2010. The Anatomy of the creative city. Industry and Innovation 17 (1), 91-111.

Collins H., 2014. Are we all Scientific Experts now? Polity, Cambridge.

Dubois V., 2012. Le rôle des street-level bureaucrats dans la conduite de l'action publique en France. Papier de recherche, Halshs.

Durance P., Godet M. et Mousli M., 2010. Créativité et innovation dans les territoires. La Documentation française, Paris.

Ewango Chatelet A., 2017. Les dynamiques intrapreneuriales des innovations pédagogiques à l'Université : une approche contextuelle. Thèse de doctorat soutenue le 23 mai 2017 à l'Université de Versailles Saint Quentin en Yvelines.

Favoreu C., 2008. Légitimité, portée et conditions d'application des outils d'aide à la décision stratégique au sein du secteur public : le cas du Centre National d'Études Spatiales (CNES). Politiques et management public 26 (1), 2-30.

Fischer F., Miller G. and Sidney M., eds, 2007. Handbook of Public Policy Analysis. Theory, Politics and Methods. CRCPress/Taylor and Francis, BocaRaton. 
Gibert P., 2008. Un ou quatre managements publics? Politiques et management public, 26 (3), 7-20.

Godin B., 2015. Innovation Contested, the Idea of Innovation over the Century. Routledge, London.

Gorman M.E., 2010. Trading Zones and Interactional Expertise, Creating new Kinds of Collaboration. MIT Press, Cambridge.

Greene J., 2017. Tribus morales, l'émotion la raison et tout ce qui nous sépare. Éditions Markus Haller, Genève.

Halpern C., Lascoumes P. et Le Gales P., 2014. L'Instrumentation de l'action publique, Controverses, résistance, effets. Presses de Sciences Po, Paris.

Heinich N., 2017. Des valeurs. Une approche sociologique. Gallimard, Paris.

Horvath I. et Dechamp G., 2016. Quand les pouvoirs publics favorisent la proximité pour stimuler la créativité du territoire. Gestion et Management Public 4, 139-155.

Le Coz A., 2015. Territoires hautement. Intervention aux Entretiens Territoriaux de Strasbourg. Inet, Lyon.

Lesourne. J et Randet D., 2013. La recherche et l'innovation en France. Rapport FUTURIS 2013. Odile Jacob, Paris.

Laville J-L. et Salmon A., 2015. Associations et action publique. Desclée de Brouwer, Paris.

Lawton T., Doh T. and Rajwani T., 2014. Aligning for Advantage. Oxford Press University, Oxford.

McCall. G.J., 2007. Ce que nous avons appris sur la construction nationale et la société civile. Revue internationale des sciences sociales 192, 277-284.

Mazeaud A., 2013. Produire la demande lycéenne : le budget participatif, instrument d'une politique régionale réactive aux besoins de la communauté lycéenne. Politiques et management public 30 (2),141-158.

Michel H., 2005. e-Administration, e-Government, e-Governance and the Learning City: a Typology of Citizenship Management using ICTs. The Electronic Journal of e-Government 3 (4), 213-218.

Noveck B., 2006. « Peer to Patent » : Collective Intelligence, Open Review, and Patent Reform. Harvard Journal of Law \& Technology 20 (1), 123-162.

Ortiz M., 2013. Varieties of Innovation System. The Governance of Knowledge Transfer in Europe. CampusVerlag, Franckfurt.

Pestre D., 2014. Le gouvernement des techno-sciences. La Découverte, Paris.

Revilliard A., 2016. Social Movements and the Politics of Bureaucratic Rights Enforcement : Insights from the Allocation of Disability Rights in France. Law and Social Inquiry 42 (2), 450-478.

Rival M. et Vidal O., 2013. Comptabilité et politique, des liens complexes et réciproques. La Revue des Sciences de Gestion Direction et gestion 263-264, 15-18.

Rival M. et Sémo I., 2014. Du lobbying à la co-construction du Service Public, une entreprise à la recherche de valeur publique dans le secteur de l'eau en France In M. Rival, F. Bournois et V. Chanut (eds), Intelligence économique et lobbying, au prisme des valeurs publiques, ESKA, Paris, 249-272.

Rival M. et Chanut V., 2015. Les stratégies politiques des organisations : de nouvelles perspectives pour la recherche en gestion. Revue Française de Gestion 252, 71-88.

Rosanvallon P., 2008. La légitimité démocratique, impartialité, réflexivité, proximité. Le Seuil, Paris.

Ruano-Borbalan J.C., ed, 2014. les voies de la construction des savoirs légitimes In Jaeger M. (dir), La recherche en travail social, Dunod, Paris.

Ruano-Borbalan J.C., ed, 2017. Science, research and Society : the Impact of Innovation and Standardisation of Knowledge Production. Journal of Innovation, Economics and Management 22, 3-8.

Scharpf F.W., 2010. Community and Autonomy, Institutions, Policies and Legitimacy in Multilevel Europe. Campus Verlag, Frankfurt/New-York,

Warin P., 1999. Les « ressortissants » dans les analyses des politiques publiques. Revue française de science politique 49 (1), 103-121.

Weller J.M. et Pallez F., 2017. Les formes d'innovation publiques par le design, un essai de cartographie. Sciences du design 5, 32-51.

Zittoun P., 2013. La fabrique politique des politiques publiques. Une approche pragmatique de l'action publique. Presses de sciences Po, Paris. 